\title{
La dystrophie myotonique: II nuptialité, fécondité et transmission
}

\author{
du gène
}

\author{
Suzanne Veillette, Michel Perron et Jean Mathieu
}

\begin{abstract}
RÉSUMÉ: Une enquête sociologique, menée auprès d'un échantillon représentatif $(N=218)$ de la population atteinte de dystrophie myotonique (DM), permet d'analyser la relation entre certains comportements démographiques des malades (nuptialité, fécondité) et les mécanismes de la transmission du gène délétère. Les résultats démontrent une très nette différenciation entre les comportements nuptiaux des femmes et des hommes atteints de DM. Alors que l'éligibilité au mariage des hommes a considérablement diminué, les femmes atteintes continuent à se marier jeunes et dans une proportion presque équivalente à la population féminine du Saguenay-Lac-Saint-Jean. L'enquête fait également ressortir que la fécondité moyenne des individus mariés au Saguenay-Lac-Saint-Jean surpasse la fécondité observée dans les populations atteintes repérées dans d'autres pays. Nous observons également une surfécondité masculine. Ce fait démographique explique la surreprésentation de transmetteurs masculins constatée aujourd'hui. Cependant si les tendances actuelles se maintiennent, ce sont les femmes surtout qui risquent de transmettre la DM aux générations futures. De telles conclusions permettent d'illustrer comment la transmission et la dispersion d'un gène dominant suivent des itinéraires qui ne doivent pas être dissociés de l'ensemble des caractéristiques socio-culturelles. Elles fournissent également des pistes utiles dans l'élaboration de certains programmes préventifs.
\end{abstract}

\begin{abstract}
Myotonic Dystrophy: II Marriage, Fertility and Gene Transmission. A sociological study, using a representative sample $(\mathrm{N}=218)$ of the myotonic dystrophy population, made possible an analysis of the relationship between certain demographic characteristics (marriage, fertility) and the mechanisms involved in the transmission of the deleterious gene. The results show a clear differentiation between the marriage rate of women and men affected by myotonic dystrophy. Whereas men show a considerable decline in marriage eligibility, women continue to marry at a young age and in a proportion almost equal to that of the unaffected population. The study also indicates that the average fertility rate among married patients in the Saguenay-Lac-Saint-Jean exceeds the fertility rate reported from affected populations found in other countries. Our study shows an above-normal male fertility rate. This demographic fact explains the overrepresentation of male transmitters noted in the affected population. However, if present trends remain unchanged, women will be more likely to transmit myotonic dystrophy to future generations. These conclusions illustrate how the transmission and spread of a dominant gene follow a pattern that cannot be dissociated from the socio-cultural characteristics taken as a whole, particularly demographic characteristics. They also provide us with useful avenues for setting up future prevention programmes.
\end{abstract}

Can. J. Neurol. Sci. 1989; 16:114-118

La dystrophie myotonique (DM) est une génopathie transmise selon le mode autosomal dominant. Un tel mode de transmission peut provoquer une prévalence très élevée dans une population lorsque certaines conditions y sont associées. Compte tenu de la très forte prévalence de cette affection au Saguenay-Lac-Saint-Jean (SLSJ), Québec (196 cas pour 100,000 habitants, en 1986), ${ }^{\prime}$ il importe de rechercher les facteurs particuliers qui peuvent être associés à un tel phénomène endémique.

Une attention particulière doit être portée, d'une part, à l'étude de certains mécanismes connus en génétique des populations (effet fondateur, sélection naturelle, échanges migratoires) $)^{2}$ et, d'autre part, à l'analyse des mécanismes d'ac- croissement naturel (nuptialité, fécondité) de la sous-population atteinte.

Notre intérêt pour la nuptialité et la fécondité des malades a donné lieu au questionnement suivant. Les individus atteints de DM ont-ils des comportements de nuptialité et de fécondité différents des individus non atteints? Les comportements de nuptialité et de fécondité des femmes atteintes sont-ils différents de ceux des hommes atteints? Le gène DM est-il transmis par une proportion équivalente d'hommes et de femmes?

Les publications médicales se sont intéressées à la nuptialité de ces malades ${ }^{3}$ et ont discuté largement de leur fécondité. Différents auteurs soulignent leur sous-fécondité relative ${ }^{3-5}$

Sociologue, professeure au département de Sciences humaines, CEGEP de Jonquière (SV); Sociologue, professeur au département de Travail social, CEGEP de Jonquière (MP); directeur de la Clinique de dystrophie musculaire, Hôpital de Chicoutimi (JM)

Tirés à part: Groupe ECOBES, CEGEP de Jonquière, 2505, rue St-Hubert, Jonquière, Québec, Canada G7X 7W2 
qu'ils attribuent tantôt à l'atrophie testiculaire observée chez les hommes atteints, ${ }^{4-5}$ tantôt aux problèmes de grossesse et aux avortements spontanés chez les femmes atteintes. ${ }^{5}$ On a observé également un écart entre la fécondité des femmes mariées et celle des hommes mariés. ${ }^{3-5}$ Toutefois, des recherches moins récentes (Bell, Thomasen) ${ }^{6}$ ont observé chez les individus atteints, une fécondité élevée et tout à fait comparable à celle des individus non atteints des mêmes familles.

Le présent article s'intéresse aux comportements démographiques de tels malades. Après avoir analysé leur nuptialité et leur fécondité, nous établirons s'il y a une surreprésentation de transmetteurs paternels ou maternels du gène DM au SLSJ.

\section{Matériel et MÉthodes}

C'est à l'aide des données d'une enquête sociologique menée auprès d'un échantillon représentatif $(\mathrm{N}=218)$ de la population atteinte de DM au SLSJ $(\mathrm{N}=462)$, que nous pouvons réaliser l'analyse des comportements nuptiaux et féconds. La méthodologie de notre enquête et la stratégie d'échantillonnage ont déjà été exposées dans la première partie de ce texte publiée dans le présent numéro. L'analyse de la transmission du gène DM a été effectuée à propos des malades pour lesquels l'information nécessaire était disponible en 1983, c'est-à-dire 385 cas.

Les malades sont tous connus de la Clinique de dystrophie musculaire de l'Hôpital de Chicoutimi et sont atteints d'une forme symptomatique de cette génopathie. ${ }^{1}$

\section{RÉSUltats et Discussion}

\section{Nuptialité}

Pour étudier les comportements nuptiaux des individus malades, nous avons considéré leur déclaration d'état matrimonial et leur âge au premier mariage. Au moment de l'enquête, on constate une différence statistiquement significative entre l'état matrimonial déclaré des femmes et des hommes atteints âgés de 15 ans et plus $\left(X^{2}=18,68 ; p=0,001\right)$. Chez les hommes, on observe une très forte propension au célibat $(65,8 \%)$, le tiers seulement $(34,2 \%)$ vivant ou ayant vécu maritalement. Chez les femmes, on découvre un profil plus habituel: la majorité $(64,4 \%)$ sont mariées ou l'ont été ou vivent maritalement, le tiers $(35,6 \%)$ demeurent encore célibataires. Selon le sexe, les proportions d'individus célibataires ou mariés sont donc totalement inversées.
Un examen de la nuptialité, en tenant compte de l'âge des individus, (figure 1) montre que chez les hommes, une très forte proportion des moins de 45 ans est célibataire $(85,7 \%)$ alors que chez les femmes, cette proportion est de $46,2 \%$. Parmi les individus âgés de 35 ans et moins, le pourcentage de célibataires s'élève à $90,7 \%$ chez les hommes comparativement à $58,7 \%$ chez les femmes. Toutes ces différences sont statistiquement significatives. Par contre, les hommes et les femmes de 55 ans et plus, déclarent des états matrimoniaux presque semblables. (Précisons qu'il n'y a pas homogamie des mariages et que dans cette figure les hommes ne sont pas les conjoints des femmes).

Lorsque nous considérons l'âge au premier mariage, nous découvrons que le calendrier de nuptialité constitue un autre indicateur de la réduction de l'éligibilité au mariage des hommes atteints. Ils se sont mariés à un âge plus avancé que les femmes (27,5 ans en moyenne comparativement à 22,0 ans en moyenne). Cette différence est statistiquement significative (t-test $=5,14 ; p=0,001)$. Un phénomène important est observé: au cours des générations, les hommes ont retardé de plus en plus leur mariage alors que les femmes ont manifesté une relative constance dans leurs comportements. Pour les promotions de mariages contractés avant 1961, la moyenne d'âge des hommes est de 25,5 ans (écart-type $=3,0$ ans) comparativement à 30,1 ans (écart-type $=8,2$ ans) pour les promotions de 1961 à 1982. Au cours des mêmes périodes, chez les femmes atteintes, la moyenne d'âge au premier mariage est passée de 21,6 ans (écart-type $=3,0$ ans) à 22,3 ans (écart-type $=3,5$ ans). De plus, seules des femmes atteintes ont eu tendance à se marier avant l'âge de 20 ans; aucun homme ne s'est marié avant 20 ans (soit 0 sur 39 ), comparativement à $28,4 \%$ chez les femmes (soit 19 sur 67).

Nous retenons de cette analyse que les femmes atteintes de cette génopathie ont toujours eu tendance à se marier plus jeunes que les hommes, quelles que soient les promotions de mariages considérées. Les comparaisons avec les données québécoises démontrent des écarts bien différents selon qu'il s'agit de femmes ou d'hommes atteints de cette maladie. Alors que les femmes atteíntes de DM se sont toujours mariées plus jeunes que les Québécoises, les hommes atteints ont eu tendance, à partir de 1961 , à se marier plus tard que les Québécois. ${ }^{7}$ Ils ont même eu tendance à retarder de plus en plus leur mariage avec le temps.

Comparés aux individus répertoriés dans les publications médicales, les malades saguenéens se marient dans une propor-

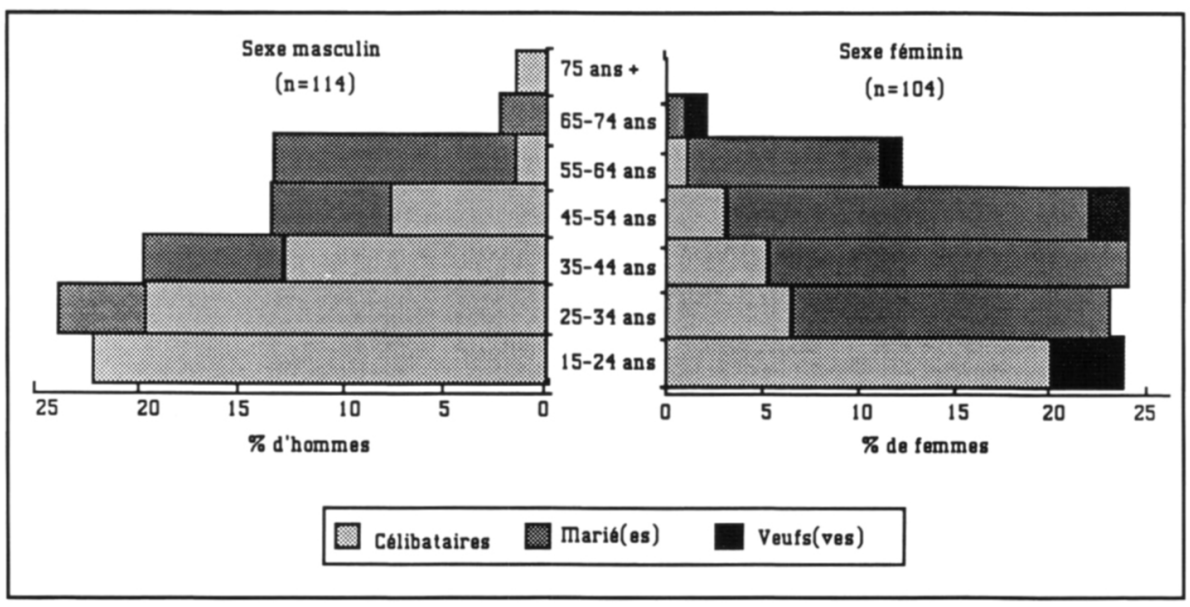

Figure 1-Profil âge/sexe des individus de 15 ans et plus atteints de DM, selon l'état matrimonial. 
tion relativement équivalente. 5 Par ailleurs, la population atteinte de DM déclare une sous-nuptialité par rapport à la population régionale $(41,3 \%$ comparativement à $61,1 \%)$. Les hommes atteints se particularisent puisqu'ils sont deux fois moins nombreux que leurs homologues saguenéens à vivre maritalement. ${ }^{8}$ D'ailleurs, pareille sous-nuptialité masculine, a déjà été relevée par Klein. ${ }^{3}$ Par contre, la nuptialité des femmes atteintes n'est que légèrement inférieure à celle des Saguenéennes.

Plusieurs questions surgissent alors. Les hommes malades, aujourd'hui âgés de plus de 45 ans, auraient-ils vraiment profité d'un plus grand degré d'éligibilité au mariage? Les contraintes culturelles (au sens large) imposées à cette génération d'hommes avaient-elles un caractère moins sélectif que celui constaté chez les générations récentes? La même maladie se manifestait-elle avec la même sévérité il y a trente ou quarante ans? Une observation longitudinale chez les générations récentes, de la relation entre l'état matrimonial et le degré d'atteinte, pourrait fournir éventuellement quelques éléments de réponse.

\section{Fécondité}

Avant d'examiner si les comportements féconds sont différentiels selon le sexe de l'individu atteint, il importe de souligner d'abord que les individus mariés au SLSJ ont été plus féconds que les autres malades observés dans d'autres pays. Nous observons au tableau 1 que la fécondité moyenne des individus mariés dans la région à l'étude (3,25 enfants) surpasse largement celle relatée par différents auteurs ${ }^{5}$ (les moyennes oscillent entre 1,90 enfant et 2,70 enfants).

Les couples touchés par la DM se sont comportés comme les membres de leur région d'appartenance. ${ }^{9}$ Les individus atteints que nous avons interviewés sont presque tous nés dans la région du SLSJ $(98,6 \%)$. Ils appartiennent à une communauté extrêmement homogène sur le plan culturel (de race blanche, francophone et de confession catholique) et cette homogénéité culturelle a été constante dans le temps. De plus, la dynamique de la croissance de la population régionale «s'explique avant tout par le maintien, jusque vers 1960 , d'une fécondité très élevée». ${ }^{10}$ La population régionale a adopté un régime de fécondité contrôlée une décennie plus tard que ne l'ont fait les Québécois en général. Par conséquent, «les indices de fécondité (générale ou légitime) y sont toujours plus élevés que dans l'ensemble du Québec entre 1852 et 1971 ". ${ }^{10}$ Pour la période plus récente, nous observons les mêmes tendances: l'indice synthétique de fécondité est relativement plus élevé dans cette région que celui observé dans l'ensemble du Québec. ${ }^{7}$

Le tableau 1 révèle de plus que la fécondité moyenne des individus malades au SLSJ, qui se sont mariés, est statistiquement différente selon le sexe ( $t$-test $=2,16 ; p=0,03$ ). Les hommes ont eu en moyenne 4,18 enfants alors que les femmes atteintes ont eu en moyenne 2,70 enfants. Dans les publications médicales, l'écart observé entre la fécondité des femmes et celle des hommes mariés atteints est relativement faible, et il ne va pas toujours dans le même sens. Tantôt, on constate une légère surfécondité masculine (Klein), tantôt on constate une légère surfécondité féminine (Harper). Un phénomène important apparaît donc dans la région à l'étude: parmi les malades qui se sont mariés, les hommes présentent une fécondité plus vigoureuse que les femmes.

Toutefois, lorsque nous considérons, au tableau 1, la fécondité moyenne générale des individus atteints, qu'ils soient mariés ou célibataires (1,59 enfant), les écarts s'estompent par rapport aux moyennes relevées chez Thomasen (1,50 enfant) et chez Caughey $(1,60$ enfant) mais ils persistent par rapport à la moyenne relatée par Klein (1,01 enfant). Notons que nous comparons des décennies et des pays différents; ce sont des différences non négligeables en matière de fécondité et de nuptialité.

Une telle analyse de la fécondité générale, selon le sexe, révèle qu'au Saguenay-Lac-St-Jean, les femmes atteintes ont eu légèrement plus d'enfants (1,76 enfant) que les hommes atteints (1,43 enfant). Cette différence n'est pas statistiquement significative mais elle indique bien l'importance du phénomène mis en évidence précédemment, soit la sous-nuptialité des hommes atteints de DM.

De tels résultats peuvent questionner certaines analyses qui ont associé la fécondité réduite des hommes à certains symptômes spécifiques, notamment l'atrophie testiculaire. La tendance observée chez les hommes à demeurer célibataires et à retarder l'âge au mariage suffit à expliquer, du moins dans la région étudiée, la réduction de leur fécondité.

L'appartenance des individus atteints à un milieu socioéconomique faible ${ }^{11}$ peut opérer des contraintes qui pèsent davantage pour les hommes que pour les femmes sur les mécanismes de choix du conjoint. ${ }^{12-14}$ Nous savons de plus que la société saguenéenne s'est urbanisée et industrialisée à partir de la décennie 1930. Ce phénomène a pu diminuer l'habilité des hommes atteints à s'introduire sur le marché du travail rémunéré et, par voie de conséquence, réduire leurs chances de se marier. Une société urbaine et industrialisée peut imposer des con-

Tableau 1: Fécondité moyenne des individus atteints de DM, selon le statut matrimonial et le sexe

\begin{tabular}{|c|c|c|c|c|c|c|c|c|c|}
\hline \multirow[b]{2}{*}{ Auteurs } & & \multicolumn{4}{|c|}{ Individus mariés } & \multicolumn{4}{|c|}{ Individus mariés et célibataires } \\
\hline & & ( $\mathrm{N}$ cas) & Hommes & Femmes & Total & ( $N$ cas) & Hommes & Femmes & Total \\
\hline Klein & (1958) & (132) & 2,30 & 1,50 & 1,90 & $(251)$ & 1,05 & 0,94 & 1,01 \\
\hline Caughey & (1963) & - & - & - & 2,00 & - & - & - & 1,60 \\
\hline Harper & (1979) & - & 1,73 & 2,09 & 1,92 & - & - & - & - \\
\hline Écobes & (1983) & $(106)^{\prime}$ & 4,18 & 2,70 & 3,25 & $(218)^{2}$ & 1,43 & 1,76 & 1,59 \\
\hline
\end{tabular}

Source: Harper, 1979: 229. 
traintes qui sont extrêmement différentes de celles qui sont propres à une société rurale.

Même si elles échappent à la conscience des individus atteints, les contraintes sociales semblent donc jouer un rôle important dans leurs stratégies matrimoniales et de fécondité. Nous pouvons d'ailleurs observer les répercussions de ces stratégies sur la transmission parentale (paternelle ou maternelle) du gène délétère.

\section{Transmission paternelle ou maternelle de la DM}

La transmission parentale (paternelle ou maternelle) de la DM varie selon le groupe d'âge d'appartenance de l'individu atteint (tableau 2). Nous constatons une surreprésentation de transmetteurs masculins, $57,1 \%$ des cas ayant reçu le gène délétère de leur père et $42,9 \%$ de leur mère. Une telle différence par rapport au résultat théoriquement attendu ( $50 \%$ pour chacun des sexes) peut se prêter à un questionnement, d'autant plus que ces proportions se sont beaucoup modifiées avec le temps. En effet, la proportion des transmetteurs paternels ou maternels varie selon le groupe d'âge d'appartenance de l'individu atteint $\left(X^{2}=23,54 ; p=0,001\right)$. Les cas appartenant aux groupes plus âgés (35 ans et plus) ont reçu le gène DM de leur père dans une proportion de 2 sur 3 alors que cette proportion est légèrement inférieure à 1 sur 2 pour les cas plus jeunes (34 ans et moins). Chez le groupe des 55 ans et plus (individus nés avant 1928), nous retrouvons la proportion attendue pour une maladie autosomale dominante. Ce qui doit retenir l'attention, c'est surtout la modification importante dans le temps de la proportion des transmetteurs masculins ou féminins. Chez les individus atteints âgés de 45 à 54 ans, c'est le père qui a transmis la maladie dans $77,8 \%$ des cas alors que chez les plus jeunes cas (14 ans et moins) c'est leur mère, dans $59,3 \%$ des cas.

La proportion de transmetteurs masculins observée au Saguenay $(57,1 \%)$ se compare à celle observée en Suisse $(60,9 \%)^{3}$ ou au Danemark $(55.6 \%)^{6}$ aucune de ces différences n'étant statistiquement significative. Harper est le seul auteur à avoir observé une proportion équivalente de transmetteurs masculins et féminins. Pour expliquer les différences constatées entre sa distribution et celles des autres auteurs, Harper fait intervenir le fait de considérer ou non, la forme congénitale de la maladie. Compte tenu de la mortalité néo-natale élevée chez les cas atteints de cette forme de dystrophie et en raison du fait qu'elle est transmise presque exclusivement par les femmes atteintes (plus de $95 \%$ des cas selon Harper) ${ }^{5}$, une surreprésen-

Table 2: Transmission parentale de la DM selon l'âge de l'individu atteint

\begin{tabular}{lcccc}
\hline \hline Groupes d'âge & Paternelle & Maternelle & Total & $\begin{array}{r}\text { Nombre de } \\
\text { cas }\end{array}$ \\
\hline $0-14$ ans & 40,7 & 59,3 & 100,0 & 27 \\
$15-24 "$ & 50,0 & 50,0 & 100,0 & 80 \\
$25-34 "$ & 50,6 & 49,4 & 100,0 & 81 \\
$35-44 "$ & 68,9 & 31,1 & 100,0 & 90 \\
$45-54 "$ & 77,8 & 22,2 & 100,0 & 54 \\
55 ans et plus & 45,3 & 54,7 & 100,0 & 53 \\
\hline Total \% & 57,1 & 42,9 & 100,0 & 385 \\
\hline
\end{tabular}

$\chi^{2}=23,54 ; \mathrm{p}=0,001$. tation de transmetteurs masculins pourrait facilement apparaître.

Pour expliquer la forte diminution de transmetteurs masculins observée au SLSJ, nous devons référer à notre analyse de la nuptialité et de la fécondité de tels malades. La réduction du degré d'éligibilité au mariage des hommes atteints et la modification importante qu'ils ont opérée relativement au calendrier de leur nuptialité expliquent en bonne partie, selon nous, la diminution des transmetteurs masculins. C'est sans doute le haut taux de nuptialité des hommes âgés de 55 ans et plus, combiné à leur forte fécondité, qui expliquent qu'une surreprésentation $(57,1 \%)$ des transmetteurs masculins soit encore observable aujourd'hui.

\section{CONCLUSION}

Les comportements nuptiaux et féconds différentiels selon le sexe permettent d'expliquer, selon nous, les modifications importantes observées dans la transmission parentale (paternelle ou maternelle) de la DM. Si dans le passé, une majorité des malades ont reçu le gène $\mathrm{DM}$ de leur père, aujourd'hui une plus forte proportion le reçoivent de leur mère. Le très faible degré d'éligibilité au mariage des hommes (particulièrement les moins de trente-cinq ans) est certainement l'un des facteurs les plus importants à retenir.

Si les tendances actuelles se maintiennent, une surreprésentation de transmetteurs féminins sera probablement observée au cours des prochaines générations. De plus, la sous-nuptialité croissante des hommes atteints, ajoutée aux problèmes typiques liés à la fécondité des femmes atteintes (avortements spontanés, mortinatalité) pourrait limiter sensiblement l'accroissement naturel de la sous-population atteinte. Une telle conclusion doit cependant être interprétée en tenant compte du fait que notre étude n'a pas considéré les comportements des individus atteints de la forme asymptomatique de DM.

Nos résultats illustrent comment la transmission du patrimoine héréditaire est reliée à certains facteurs socio-culturels. La transmission et la dispersion d'un gène dominant suivent des itinéraires et des canalisations qui ne peuvent être dissociés de l'ensemble des faits sociaux et plus particulièrement des comportements démographiques. ${ }^{15}$ Les résultats peuvent également servir de guide dans l'élaboration de certains programmes préventifs.

\section{REMERCIEMENTS}

Cet article est le résultat de travaux de recherche réalisés par le groupe ECOBES du CEGEP de Jonquière. Cette recherche a bénéficié du soutien financier du Fonds pour la Formation de Chercheurs et l'Aide à la Recherche (FCAR), programme ACSAIR, et de la Fondation Asselin de Jonquière.

Nous remercions G. Hébert, B. Ortmann, C. Prévost et M. De Braekeleer qui ont commenté une version préliminaire de cet article.

\section{RÉFÉRENCES}

1. Mathieu J, Simard M, De Braekeleer M, et al. Partial syndrome of myotonic dystrophy: clinical presentation and follow-up. Can J Neurol Sci 1989, 99-103.

2. Jacquard A. Génétique des populations humaines. Paris: Presses Universitaires de France 1974.

3. Klein D. La dystrophie myotonique (Steinert) et la myotonie congénitale (Thomsen) en Suisse. Etude clinique, génétique et démographique. J gén hum 1958; 7: 28-29. 
4. Caughey JE, Myrianthopoulos NC. Dystrophia myotonica and related disorders. Springfield: Charles C Thomas 1963; 198200.

5. Harper PS. Myotonic Dystrophy. Philadelphia: W.B. Saunders 1979; 115-126, 229.

6. Thomasen E. Myotonia. Denmark: Aarhuus Stiftsbogtrykkerie 1948; 169-170.

7. Bureau de la statistique du Québec. Démographie québécoise: passé, présent, perspectives. Québec 1983; 119-175.

8. Statistique Canada. Recensement de la population. Ottawa: Approvisionnements et Services 1982; catalogue 93-917.

9. Perron M, Veillette S, Desbiens F, Mathieu J. Comportements socio-démographiques des individus atteints de dystrophie myotonique. Cahiers québécois de démographie 1986; 15: 75110.

10. Pouyez C, Lavoie Y, Bouchard G, et al. Les Saguenayens. Introduction à l'histoire des populations du Saguenay, XVIe -
XXe siècles. Québec: Presses de l'Université du Québec 1983; 282-329.

11. Veillette S, Perron M, Desbiens F. La dystrophie myotonique: étude épidémiologique et socio-géographique au Saguenay-Lac-SaintJean. Jonquière: Cégep de Jonquière, rapport de recherche 1986.

12. Girard A. Le choix du conjoint. Une enquête psycho-sociologique en France. Paris: Presses universitaires de France 1964.

13. Vu Tien Khang J, Sevin A. Choix du conjoint et patrimoine génétique. Étude de quatre villages du Pays de Sault de 1740 à nos jours. Toulouse: Editions du CNRS 1977.

14. Bourdieu P. Les stratégies matrimoniales dans le système de reproduction. Annales ESC 1972; 4-5: 1105-1125.

15. Segalen M, Jacquard A. Isolement sociologique et isolement génétique. Population 1973; 3: 551-570. 